\title{
The metabolic effects of multi-trace elements on parenteral nutrition for critically ill pediatric patients: a randomized controlled trial and metabolomic research
}

\author{
Qingti Tan $^{1 \#}$, Yu Wang ${ }^{2,3 \#}$, Guoying Zhang ${ }^{1 \#}$, Bin Lu' ${ }^{1}$, Tao Wang ${ }^{1}$, Tao Tao ${ }^{1}$, He Wang ${ }^{4 \#}$, Hua Jiang ${ }^{2,3}$, \\ Wei Chen ${ }^{5}$
}

${ }^{1}$ Pediatric Intensive Care Unit, Chengdu Women's and Children's Central Hospital, School of Medicine, University of Electronic Science and Technology of China, Chengdu, China; ${ }^{2}$ Institute for Emergency and Disaster Medicine, Sichuan Provincial People's Hospital, University of Electronic Science and Technology of China, Chengdu, China; ${ }^{3}$ Institute for Emergency and Disaster Medicine, Chinese Academy of Science Sichuan Translational Medicine Research Hospital, Chengdu, China; ${ }^{4}$ School of Pharmacy, Shanghai University of Traditional Chinese Medicine, Shanghai, China; ${ }^{5}$ Department of Clinical Nutrition, Department of Health Medicine, Peking Union Medical College Hospital, Chinese Academy of Medical Sciences and Peking Union Medical College, Beijing, China

Contributions: (I) Conception and design: W Chen, H Jiang; (II) Administrative support: H Wang; (III) Provision of study materials or patients: Q Tan, G Zhang, B Lu, T Wang, T Tao; (IV) Collection and assembly of data: Q Tan, Y Wang, H Wang; (V) Data analysis and interpretation: Y Wang, Q Tan; (VI) Manuscript writing: All authors; (VII) Final approval of manuscript: All authors.

\#These authors contributed equally to this work.

Correspondence to: Wei Chen. Department of Clinical Nutrition, Department of Health Medicine, Peking Union Medical College Hospital, Chinese Academy of Medical Sciences and Peking Union Medical College, Beijing, China. Email: chenw@pumch.cn; Hua Jiang. Institute for Emergency Medicine and Disaster Medicine, Sichuan Provincial People's Hospital, University of Electronic Science and Technology of China, Chengdu, China. Email: cdjianghua@qq.com.

Background: We investigated the efficacy and metabolic dose-effect of multi-trace element injection I [MTEI-(I)] for severe pediatric patients via a parallel, randomized control study.

Methods: The inclusion criteria were as follows: (I) patients who required parenteral nutrition (PN) due to various diseases, and were expected to receive $\mathrm{PN}$ for $>5$ days; (II) patients aged $<18$ years; (III) patients with no serious cardiac, hepatic, renal, or pulmonary dysfunction; and (IV) patients with an established central venous pathway. Enrolled patients were randomly assigned into two groups using sequentially numbered, sealed, opaque envelopes: Group A (low-dose group) received MTEI-(I) at $1 \mathrm{~mL} / \mathrm{kg} / \mathrm{d}$, and Group B (highdose group) received MTEI-(I) at $2 \mathrm{~mL} / \mathrm{kg} / \mathrm{d}$, up to a maximum dose of $15 \mathrm{~mL} / \mathrm{d}$. The concentrations of manganese $(\mathrm{Mn})$, copper $(\mathrm{Cu})$, zinc $(\mathrm{Zn})$, and selenium $(\mathrm{Se})$ were detected. The following indexes were measured after 5 days of treatment (T5): $\beta$-oxidation of very-long-chain fatty acids, arginine and proline metabolism, pentose phosphate metabolism, ketone body metabolism, citric acid cycle, purine metabolism, caffeine metabolism, and pyruvate metabolism. The participants, care givers, and data analysis staff were blinded to the group assignment.

Results: Overall, at T5, Mn and Cu levels were decreased, while $\mathrm{Zn}$ and Se levels were increased. The increase of $\mathrm{Zn}$ levels (A: $0.170 \pm 0.479$ vs. B: $0.193 \pm 0.900)$ and decrease of Cu levels (A: $-0.240 \pm 0.382$ vs. B: $-0.373 \pm 0.465)$ of patients in Group B $(n=22)$ were significantly higher than those in Group A ( $n=18)$. At T5, the $\beta$-oxidation of very-long-chain fatty acids, arginine and proline metabolism, pentose phosphate metabolism, ketone body metabolism, citric acid cycle, purine metabolism, caffeine metabolism, and pyruvate metabolism were variably decreased $(\mathrm{P}<0.05)$ in Group B compared to Group A.

Conclusions: Our results suggested that the high-dose administration of MTEI-(I) is safe for severe pediatric patients, and may alleviate inflammation and antioxidation, relieve hyperactivity caused by stress, and improve tissues-based hypoxia and renal function.

Trial Registration: Chinese Clinical Trial Registry ChiCTR2100052198. 
Keywords: Multi-trace element injection I [MTEI-(I)]; pediatric; metabolomics; parenteral nutrition (PN); intensive care

Submitted Aug 26, 2021. Accepted for publication Oct 20, 2021.

doi: $10.21037 / \mathrm{tp}-21-456$

View this article at: https://dx.doi.org/10.21037/tp-21-456

\section{Introduction}

Optimal nutritional support is considered to be important for critically ill pediatric patients in the intensive care unit (ICU), since malnutrition and inadequate nutrient supply are related to worse clinical outcomes (1). To improve nutritional status and cure rates of associated conditions, clinical guidelines (2) have recommended that total or supplementary parenteral nutrition (PN) should be administered to provide calories, amino acids, and other nutrients in situations where there is unavailable or insufficient enteral nutrition (EN).

Patients receiving PN should also receive trace elements (TEs), since failure to receive adequate TEs may lead to clinical symptoms of deficiencies (3). Low circular TEs are associated with the application of continuous renal replacement therapy, systemic inflammatory response, and cardiac surgery (4). Even subclinical TE deficiencies can be theoretically detrimental to a patient's recovery (5). However, there are few randomized clinical trials investigating the effects of nutritional supply on the clinical outcomes of critically ill pediatric patients (4). Well-designed clinical studies are urgently needed to further investigate the pediatric micronutrient requirements among pediatric critically ill patients.

Metabolomics is an emerging high-throughput research method that reflects body health or disease status (6). It provides an important area of science, namely, that exploring the effects of biometals in biological systems, with extremely high sensitivity (7). Moreover, since numerous metals are linked to biomolecules (proteins or metabolites), techniques for separation, such as liquid chromatography and capillary electrophoresis, are required in metabolomics studies (7). Using these technologies, we explored the TE deficiencies in ICU pediatric patients, and analyzed the efficacy and safety of different multi-trace element injection [MTEI-(I)] doses on the nutritional metabolism of these patients. We present the following article in accordance with the CONSORT reporting checklist (available at https://dx.doi.org/10.21037/tp-21-456).

\section{Methods}

\section{Patients}

In total, 40 critical ill patients requiring $\mathrm{PN}$ who were hospitalized at the Chengdu Women's and Children's Central Hospital from November 2017 to March 2018 were enrolled. The research protocol was approved by the ethics committee of Chengdu Women's and Children's Central Hospital (No. 2017 [21]). The study was conducted according to the guidelines of the Declaration of Helsinki (as revised in 2013).

Inclusion criteria: (I) patients requiring $\mathrm{PN}$ due to gastrointestinal failure, congenital malformation surgery of the digestive tract, other congenital malformation surgeries, gastrointestinal bleeding, and were expected to receive $\mathrm{PN}$ for $>5$ days; (II) patients aged $<18$ years; (III) patients with no serious cardiac, hepatic (hepatic function index exceeding twice the normal upper limit), renal [below chronic kidney disease (CKD) IV stage], or pulmonary dysfunction; (IV) patients where the central venous pathway (subclavian, internal jugular, femoral vein, or peripherally inserted central catheter) was established.

Exclusion criteria: (I) patients with allergies to $\mathrm{PN}$ components; (II) patients with diabetes; (IIII) patients with allergies or adverse reactions to known TEs; (IV) patients with obvious trace element deficiencies [iron $(\mathrm{Fe})$, zinc $(\mathrm{Zn})$, copper $(\mathrm{Cu})$, etc.]; $(\mathrm{V})$ patients with congenital metabolic abnormalities; (VI) patients with Fe deficient anemia.

General information, such as gender, weight, main diagnosis, vital signs, length of hospital stay, and hospitalization expenses were collected. At the same time, we also collected routine blood and biochemical data, as well as blood samples from the patients before PN, and after 1, 3, and 5 days of treatment, for TE and metabolomics studies.

\section{Study design}

This is a prospective, paralleled, randomized controlled trial. Patients were randomly assigned to low-dose MTEI(I) group or high-dose MTEI-(I) group (1:1 ratio), using a 


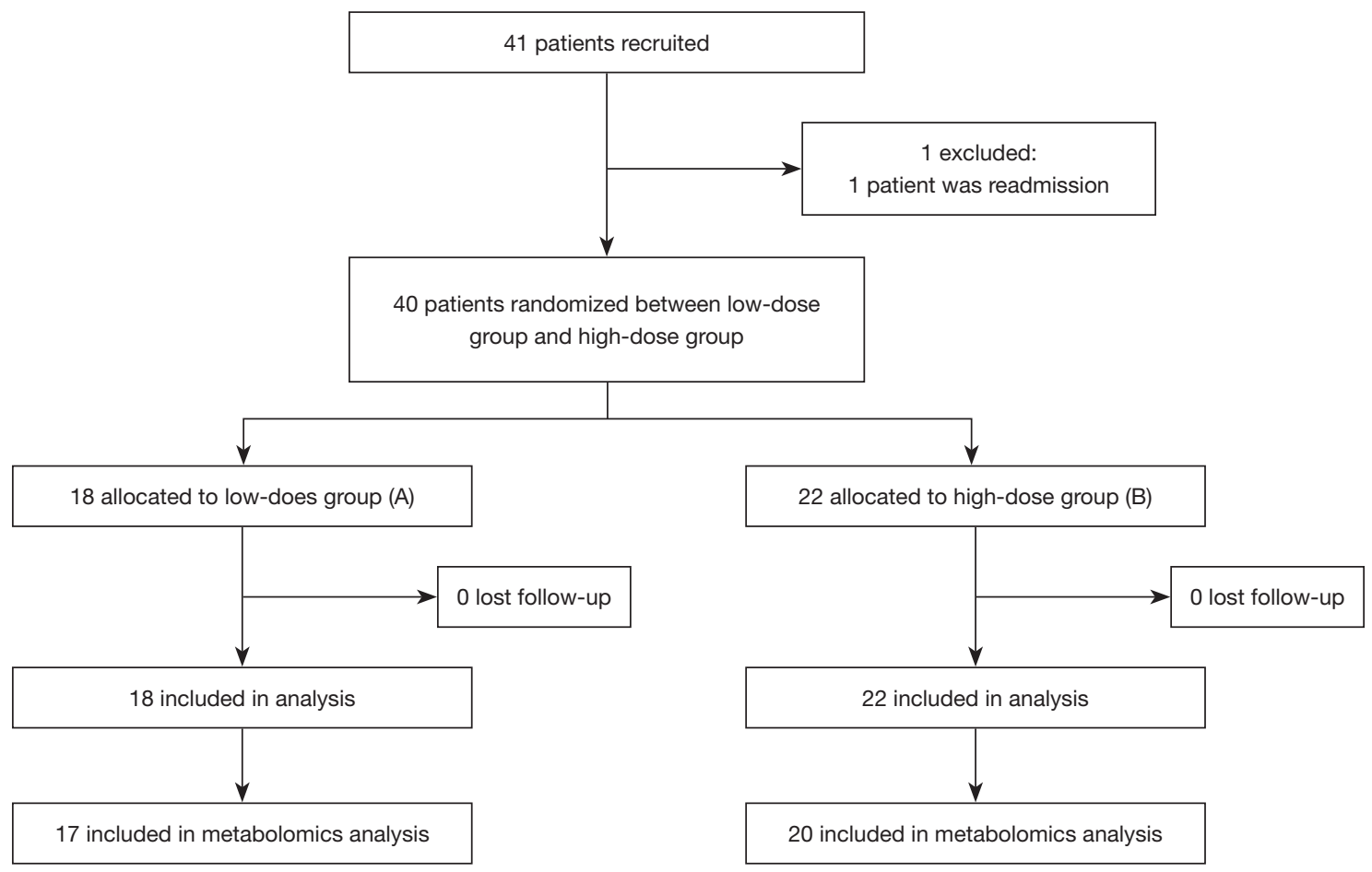

Figure 1 Patient recruitment into groups.

computerized random number generator and sequentially numbered, sealed, opaque envelopes. MTEI-(I) contains $\mathrm{Zn}(249 \mu \mathrm{g} / \mathrm{mL}), \mathrm{Cu}(20.1 \mu \mathrm{g} / \mathrm{mL}), \mathrm{Mn}(1 \mu \mathrm{g} / \mathrm{mL}), \mathrm{Se}$ $(2 \mu \mathrm{g} / \mathrm{mL})$, fluorine (F, $57 \mu \mathrm{g} / \mathrm{mL}$ ) and iodine (I, $1 \mu \mathrm{g} / \mathrm{mL}$ ). The random allocation sequence, participant inclusion, and intervention assignation were carried out by different staff. As for the sample size, all patients meeting the inclusion criteria in our hospital were included. Patients assigned to Group A were administrated MTEI-(I) at $1 \mathrm{~mL} / \mathrm{kg} / \mathrm{d}$, while those assigned to Group B were administrated MTEI-(I) at $2 \mathrm{~mL} / \mathrm{kg} / \mathrm{d}$, up to a maximum dose of $15 \mathrm{~mL} / \mathrm{d}$. The dosage standards met the requirements of the guidelines $(8,9)$ and did not exceed the maximum dose specified in the drug label. The two interventions were similar in appearance and color. Patients in both groups received basic standard of care treatment according to their clinical needs, and were given $\mathrm{PN}$ support for at least 5 consecutive days (as shown in Figure 1). The following indexes were measured after 5 days of treatment: $\beta$-oxidation of very-long-chain fatty acids, arginine and proline metabolism, pentose phosphate metabolism, ketone body metabolism, citric acid cycle, purine metabolism, caffeine metabolism, and pyruvate metabolism. Metabolic changes were the primary endpoints. The concentrations of $\mathrm{Mn}, \mathrm{Cu}, \mathrm{Zn}$, and Se were detected and as the secondary. The participants, care givers, and data analysis staff were blinded to the group assignment. All patients or statutory guardians provided informed consent.

\section{Sample collection}

After enrolment, bloods were drawn prior to PN treatment, and after 1, 3, and 5 days of PN treatment. Approximately $2 \times 2 \mathrm{~mL}$ samples were taken each time, with one sample taken in a non-anticoagulant blood tube for trace element detection, and the other into an anticoagulant tube for proton nuclear magnetic resonance $\left({ }^{1} \mathrm{H}-\mathrm{NMR}\right)$ analysis. Samples were centrifuged at 4,000 rpm for $10 \mathrm{~min}$, and the upper plasma layer was subsequently removed and stored at $-80{ }^{\circ} \mathrm{C}$.

\section{Analytical method of TE detection}

Samples were sent to the Beijing Qingxi Technology Research Institute for TE detection.

\section{Methodology}

Instruments and reagents

Inductively Coupled Plasma-Mass Spectrometry (ICP- 
MS), NexION 300 (PerkinElmer, USA); direct injection system (PerkinElmer); multi-element mixed standard solution: $10 \mathrm{mg} / \mathrm{L}$, CAS\#: Nitric acid $\left(\mathrm{HNO}_{3}\right)$ (7697-372) (PerkinElmer). Nitric acid: Suprapu (Merck KGaA, Germany). Oxygen: 99.999\%. Water: ultrapure water obtained after double treatment by UPR pure water system.

\section{Instrument conditions}

Power of RF generator: 1,600 W; plasma gas flow rate: $18 \mathrm{~L} / \mathrm{min}$; auxiliary gas flow rate $(\mathrm{Ar}): 1.2 \mathrm{~L} / \mathrm{min}$; carrier gas flow (Ar): $0.96 \mathrm{~L} / \mathrm{min}$; chamber vacuum: $1 \times 10^{-6}$; analog voltage: $-1,700 \mathrm{~V}$; pulse voltage: $850 \mathrm{~V}$; measurement method: quantitative analysis; organic sampling system conditions: oxygen flow rate of $8 \mathrm{~mL} / \mathrm{min}$; temperature of $4^{\circ} \mathrm{C}$.

\section{Sample pretreatment and preparation of standard solution}

Pre-treatment: $0.25 \mathrm{~mL}$ serum sample, added with $0.8 \mathrm{~mL}$ of $65 \% \mathrm{HNO}_{3}$ and $0.2 \mathrm{~mL}$ of $29-32 \% \mathrm{H}_{2} \mathrm{O}_{2}$, heated and digested on a heating plate at $90{ }^{\circ} \mathrm{C}$ for $3 \mathrm{~h}$, then diluted to $10 \mathrm{~mL}$ with deionized water.

Preparation of standard solution: the $10 \mathrm{mg} / \mathrm{L}$ standard mixture solution diluted to $1 \mathrm{mg} / \mathrm{L}$ for future use.

\section{Calibration curve}

The multi-element mixed standard solution was accurately pipetted, the standard curve solution was prepared, and the final concentrations were $1,5,10,20,50$, and $100 \mu \mathrm{g} / \mathrm{L}$. The prepared standard solution was tested using the organic injection system to obtain the standard curve.

In the experiment, parallel samples and spike recovery were used as the means of sample quality control to ensure the accuracy of the results.

\section{Metabolomics}

\section{Preparation of plasma samples}

After thawing at room temperature, plasma samples were centrifuged at $16,000 \mathrm{rpm}$ for $10 \mathrm{~min}$. Next, $50 \mu \mathrm{L}$ of deuterated heavy water $\left(\mathrm{D}_{2} \mathrm{O}\right)$ was added to a nuclear magnetic resonance (NMR) tube, to which $450 \mu \mathrm{L}$ of plasma was added. The sample was shaken for $2 \mathrm{~min}$ and incubated at room temperature for $10 \mathrm{~min}$ until ${ }^{1} \mathrm{H}-\mathrm{NMR}$ $(600 \mathrm{MHz})$ analysis.

\section{${ }^{1} \mathrm{H}-\mathrm{NMR}$ data collection}

A one-dimensional hydrogen spectrum was obtained after sample processing. In this study, ${ }^{1} \mathrm{H}-\mathrm{NMR}$ analysis was performed using a Bruker Avance DR ×600 MHz model (BRUKER, Germany), with a working frequency of 600.13 $\mathrm{MHz}$, equipped with a Bruker inverse broad band probe
(rIBB). The addition of $10 \% \mathrm{D}_{2} \mathrm{O}$ inhibited the solvent peak during sample preparation, and the pulse sequence (zgp) was used to inhibit the water peak during pre-saturation. All spectra were collected at room temperature [i.e., 300 Kelvin $(\mathrm{K})]$, with a spectrum width of $20 \mathrm{ppm}$, sampling points of $32 \mathrm{~K}$, and a cumulative frequency of 256 times.

\section{${ }^{1}$ H-NMR spectrum processing}

In plasma samples, the molecular nucleus of a compound resonates in a high magnetic field, and its frequency is gradually decreased. The original decay signal of the tested sample referred to our raw data (free induction decay; FID). The FID signal was imported into MestReNova software (MestreLab Research, Spain) for Fourier transformation, to generate one-dimensional ${ }^{1} \mathrm{H}-\mathrm{NMR}$ spectra. These were processed for chemical shift and automatic baseline adjustment. The convolution technique was used to minimize changes in peaks, and to ensure that larger peaks did not cover up smaller ones. Subsequently, for all plasma samples, 0-9 parts per million (ppm) segments of onedimensional hydrogen spectra were divided into $0.04 \mathrm{ppm}$ sections, and 223 chemical shift value segments were integrated to finally obtain corresponding integral values. The two-dimensional matrix was then exported in CSV format for analysis.

\section{Data preprocessing}

All data matrices were preprocessed - line normalization and standardization. Due to differences in the plasma sample dilution, concentration, test temperature, instrument working stability, and other factors during processing and measurement, ${ }^{1} \mathrm{H}-\mathrm{NMR}$ spectra from the same types of plasma samples in different batches were not completely consistent. Therefore, line normalization of data matrices was required (it was assumed that the highest peak in each ${ }^{1} \mathrm{H}-\mathrm{NMR}$ spectrum referred to the same substance with very similar content). The line normalization formula was represented as:

$$
X^{*}=\frac{X-\min }{\max -\min }
$$

\section{Spectrum data analysis}

We used the supervised pattern recognition method, Partial Least Squares-Discriminant Analysis (PLS-DA) to perform data dimension reduction. The variable importance in the projection (VIP) of the PLS-DA model, with corresponding chemical shifts, was calculated. Chemical shifts with VIP values $>1$ and $\mathrm{P}<0.05$ were selected, and corresponding 
metabolites were investigated using the human metabolome database (HMDB, https://hmdb.ca/).

\section{Statistical analysis}

All clinical data were statistically analyzed using SPSS Version 21.0 software (International Business Machines Corporation, the United States of America), and described by median (interquartile range) or mean values \pm standard deviation (SD) according to distribution type. Measurement data were first tested for data distribution type. Student's $t$-test was used for normally distributed data, and the rank-sum test was used for non-normally distributed data. The chi-square test was used for enumeration data. The statistical significance level was set at $\mathrm{P}<0.05$. The mean substitution method was used for missing clinical data.

\section{Results}

\section{Patients' status}

In total, 40 patients (ranging from 29 days to 10 years old) were enrolled into the study from November 2017 to March 2018, including 18 patients in Group A and 22 patients in Group B (as shown in Figure 1). The principal diagnoses were as follows: (I) ten patients had received fistulation surgery; (II) six patients had acute upper gastrointestinal bleeding; (III) four patients had congenital mega-colon; (IV) three patients had acute gangrenous appendicitis with perforation; (V) two patients had congenital hypertrophic pyloric stenosis; (VI) two patients had adhesive intestinal obstruction; and (VII) two patients had acute intussusception. The remaining diagnoses included the following: one patient each with Merkel diverticulitis with bleeding, necrotizing enterocolitis, acute descending colon perforation, small intestine torsion, portal hypertension syndrome, acute severe myocarditis, toxic intestinal paralysis, autotransplantation after splenectomy, congenital anal atresia and traumatic splenic rupture, and oesophageal atresia surgery.

The patients' information at admission is shown in Table 1 . No significant differences were observed between the groups in terms of gender, weight, pediatric critical illness score, vital signs, length of hospital stay, and hospitalization expenses. Similarly, we observed no significant differences in the routine blood and biochemical tests, except hemoglobin levels.

Economic aspects were also considered in this study. After comparing the length of hospital stay and hospitalization expenses between the groups, we observed that the high-dose administration of MTEI-(I) did not significantly prolong these factors for patients, suggesting minimal impact and burden on patients and their families.

\section{Changes in the general condition of patients before and after treatment}

According to TE detection results, there were no significant differences observed in patients after 1, 3, and 5 days of treatment in each group, and therefore, we focused on and analyzed the pre-treatment data (T0) as well as that after 5 days of treatment (T5). The general condition, routine bloods, and biochemistry of both groups after 5 days of treatment are shown in Table 2; no significant differences were observed between the two groups. Routine blood and biochemistry data before and after treatment were compared between the groups (Table 3). After treatment, the white blood cells (WBC), neutrophil $(\mathrm{N})$, creatinine $(\mathrm{Cr})$, total bilirubin (TB), direct bilirubin (DB), and albumin (ALB) decreased in both groups, of which $\mathrm{WBC}$ and $\mathrm{Cr}$ in Group B were significantly lower after 5 days of treatment.

\section{TE data in patients before and after treatment}

The patients' TE data before and after treatment are shown in Table 4. After 5 days of treatment, $\mathrm{Mn}$ and $\mathrm{Cu}$ decreased to different extents, whereas $\mathrm{Zn}$ and Se increased in both groups. For both $\mathrm{Zn}$ and $\mathrm{Cu}$, we observed significant differences compared to the pre-treatment levels. We also compared TE data in both groups before and after treatment (Table 5). After treatment, $\mathrm{Mn}$ and $\mathrm{Cu}$ decreased in both two groups, while the levels of $\mathrm{Zn}$ and Se increased. Compared to Group A, Zn levels in Group B increased significantly, and $\mathrm{Cu}$ in Group B decreased markedly.

\section{Differences in patient metabolomics before and after treatment}

Qualified metabonomic samples were obtained from 37 patients in both groups; 17 from Group A and 20 from Group B. We used ${ }^{1} \mathrm{H}-\mathrm{NMR}$ metabolic fingerprinting of patient plasma to distinguish patient metabolomics before treatment (T0) and after treatment (T5) (Figure 2). The VIP of chemical shift values of the metabolites at $\mathrm{T} 0$ and $\mathrm{T} 5$ are shown in Figure 3, of which those with a VIP $\geq 1$ and $\mathrm{P}<0.05$ were taken as characteristic metabolites (Tables 6,7). According to Tables 6,7, after 5 days of treatment, valine, leucine, 
Table 1 General condition of patients before treatment

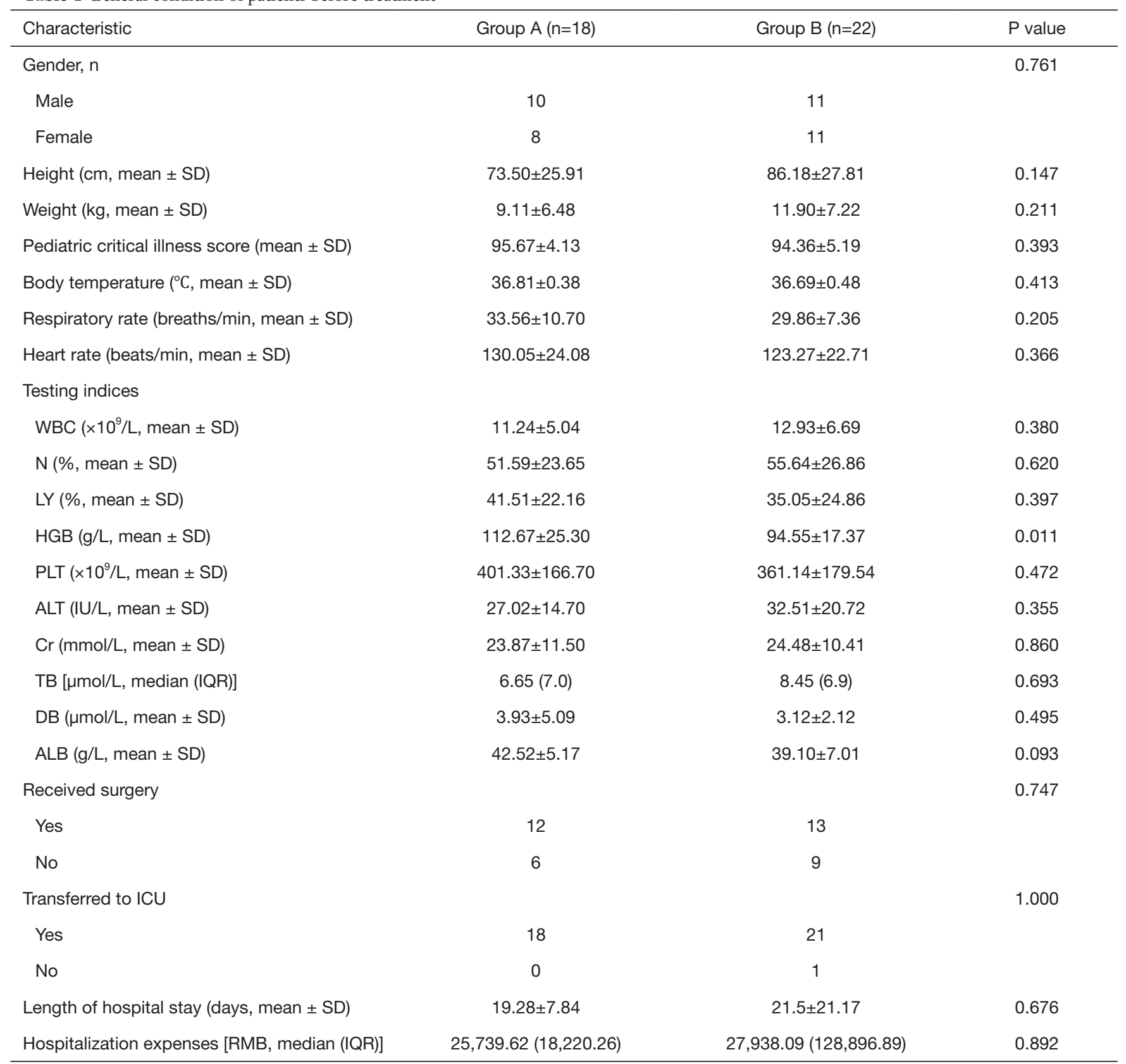

SD, standard deviation; IQR, interquartile range; WBC, white blood cell; N, neutrophile granulocyte; LY, lymphocyte; HGB, hemoglobin; PLT, platelets; ALT, alanine aminotransferase; Cr, creatinine; TB, total bilirubin; DB, direct bilirubin; ALB, albumin; ICU, intensive care unit; $\mathrm{RMB}$, renminbi.

isoleucine ( $\alpha$-ketoisovaleric acid), taurine and hypotaurine metabolism (hypotaurine), arginine and proline metabolism (phosphocreatine and glycocyamine), ketone body metabolism (acetoacetic acid and acetone), and other metabolic processes were significantly decreased.
Figure 4 shows PLS-DA analyses based on patient metabolic fingerprint spectra. From these data, we observed metabolic differences between patients in both groups. The VIP metabolite data are shown in Figure 5, for which the characteristic metabolites with a VIP $>1$ and $\mathrm{P}<0.05$ 
Table 2 General patient data after 5 days of treatment

\begin{tabular}{|c|c|c|c|}
\hline Characteristic & Group A $(n=18)$ & Group B $(n=22)$ & $P$ value \\
\hline Respiratory rate (breaths/min, mean $\pm \mathrm{SD}$ ) & $32.59 \pm 8.43$ & $29.05 \pm 7.22$ & 0.172 \\
\hline Heart rate (beats/min, mean $\pm S D$ ) & $122.18 \pm 14.01$ & $111.95 \pm 21.39$ & 0.099 \\
\hline \multicolumn{4}{|l|}{ Testing index } \\
\hline $\mathrm{N}(\%$, mean $\pm \mathrm{SD})$ & $46.35 \pm 17.59$ & $54.77 \pm 16.43$ & 0.127 \\
\hline $\mathrm{LY}(\%$, mean $\pm \mathrm{SD})$ & $42.08 \pm 14.62$ & $33.49 \pm 17.35$ & 0.103 \\
\hline $\mathrm{HGB}(\mathrm{g} / \mathrm{L}$, mean $\pm \mathrm{SD})$ & $95.98 \pm 9.79$ & $100.80 \pm 15.93$ & 0.269 \\
\hline $\mathrm{PLT}\left(\times 10^{9} / \mathrm{L}\right.$, mean $\left.\pm \mathrm{SD}\right)$ & $392.61 \pm 94.13$ & $380.50 \pm 168.32$ & 0.776 \\
\hline $\mathrm{TB}(\mu \mathrm{mol} / \mathrm{L}$, mean $\pm \mathrm{SD})$ & $7.75 \pm 8.18$ & $7.38 \pm 3.98$ & 0.852 \\
\hline $\mathrm{DB}(\mu \mathrm{mol} / \mathrm{L}$, mean $\pm \mathrm{SD})$ & $2.53 \pm 2.25$ & $2.59 \pm 1.45$ & 0.923 \\
\hline ALB $(g / L$, mean $\pm S D)$ & $35.86 \pm 4.42$ & $36.55 \pm 4.89$ & 0.644 \\
\hline
\end{tabular}

SD, standard deviation; WBC, white blood cell; N, neutrophile granulocyte; LY, lymphocyte; HGB, hemoglobin; PLT, platelets; ALT, alanine aminotransferase; Cr, creatinine; TB, total bilirubin; DB, direct bilirubin; ALB, albumin.

Table 3 Routine test data in both groups before and after treatment

\begin{tabular}{|c|c|c|c|c|c|c|}
\hline Testing index & \multicolumn{3}{|c|}{ Group A ( $n=18)$} & \multicolumn{3}{|c|}{ Group B (n=22) } \\
\hline WBC $\left(\times 10^{9} / L\right.$, mean $\left.\pm S D\right)$ & $11.24 \pm 5.04$ & $8.34 \pm 3.39$ & 0.085 & $12.93 \pm 6.69$ & $8.70 \pm 4.63$ & 0.011 \\
\hline $\mathrm{N}(\%$, mean $\pm \mathrm{SD})$ & $51.59 \pm 23.65$ & $46.35 \pm 17.59$ & 0.267 & $55.64 \pm 26.86$ & $54.77 \pm 16.43$ & 0.873 \\
\hline LY $(\%$, mean \pm SD $)$ & $41.51 \pm 22.16$ & $42.08 \pm 14.62$ & 0.901 & $35.05 \pm 24.86$ & $33.49 \pm 17.35$ & 0.754 \\
\hline $\mathrm{PLT}\left(\times 10^{9} / \mathrm{L}\right.$, mean $\left.\pm \mathrm{SD}\right)$ & $401.33 \pm 166.70$ & $392.61 \pm 94.13$ & 0.838 & $361.14 \pm 179.54$ & $380.50 \pm 168.32$ & 0.642 \\
\hline ALT (IU/L, mean \pm SD) & $27.02 \pm 14.70$ & $34.06 \pm 24.73$ & 0.335 & $32.51 \pm 20.72$ & $36.83 \pm 21.57$ & 0.505 \\
\hline $\mathrm{Cr}(\mathrm{mmol} / \mathrm{L}$, mean $\pm \mathrm{SD})$ & $23.87 \pm 11.50$ & $19.76 \pm 5.23$ & 0.056 & $24.48 \pm 10.41$ & $18.10 \pm 4.94$ & 0.007 \\
\hline TB $(\mu \mathrm{mol} / \mathrm{L}$, median (IQR)) & $6.65(4.15-11.28)$ & $4.48(3.53-7.96)$ & 0.005 & $8.45(4.75-11.68)$ & $6.55(4.8-9.9)$ & 0.112 \\
\hline
\end{tabular}

SD, standard deviation; IQR, interquartile range; WBC, white blood cell; N, neutrophile granulocyte; LY, lymphocyte; HGB, hemoglobin;

PLT, platelets; ALT, alanine aminotransferase; Cr, creatinine; TB, total bilirubin; DB, direct bilirubin; ALB, albumin.

are shown in Table 8. For Group B, $\beta$-oxidation of verylong-chain fatty acids (hexacosanoic acid), arginine and proline metabolism (phosphocreatine), pentose phosphate metabolism (D-ribose), ketone body metabolism (acetone), citric acid cycle (succinic acid), purine metabolism (adenine), caffeine metabolism (dimethylxanthine), and pyruvate metabolism (acetyl phosphate) were all decreased compared to Group A at T5. 
Table 4 Trace element data in 40 patients before and after treatment $(\mathrm{mg} / \mathrm{L})$

\begin{tabular}{lccc}
\hline Testing index & T0 & T5 & P value \\
\hline Mn (mean \pm SD) & $0.044 \pm 0.028$ & $0.035 \pm 0.053$ & 0.303 \\
Zn [median (IQR)] & $1.028(1.050)$ & $1.052(1.101)$ & 0.013 \\
Cu (mean \pm SD) & $1.700 \pm 0.601$ & $1.387 \pm 0.549$ & 0.000 \\
Se [median (IQR)] & $0.086(0.039)$ & $0.095(0.050)$ & 0.089 \\
\hline
\end{tabular}

$\mathrm{SD}$, standard deviation; IQR, interquartile range; Mn, manganese; $\mathrm{Zn}$, zinc; Cu, copper; Se, selenium.

Table 5 Patients data in both groups before and after treatment* $(\mathrm{mg} / \mathrm{L})$

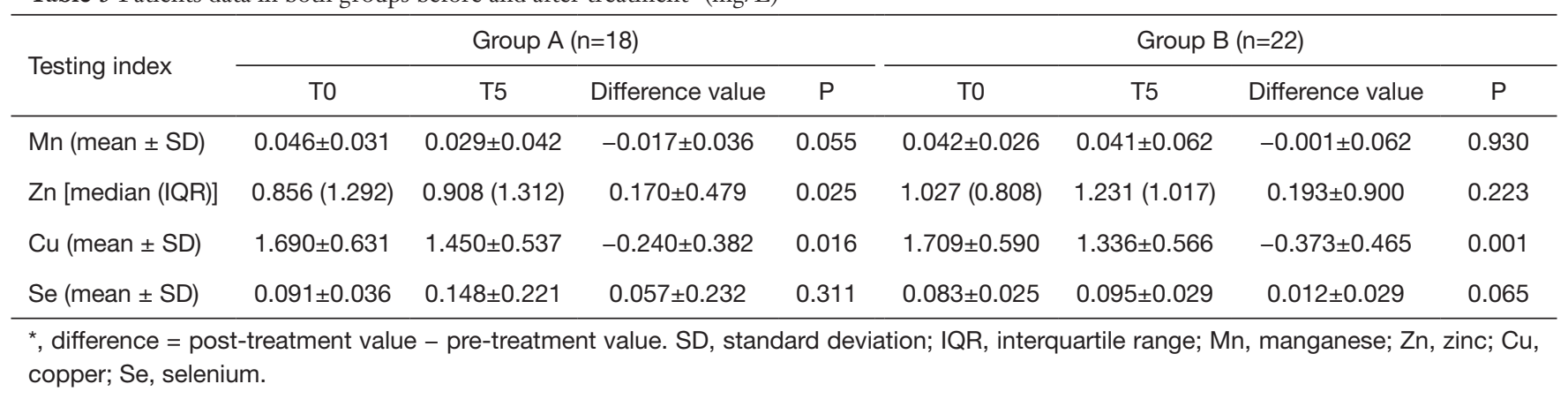

A Samples/scores plot of data

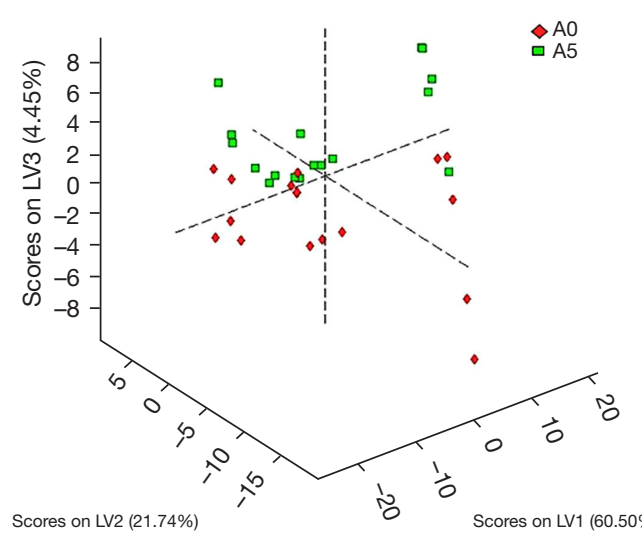

B

Samples/scores plot of data

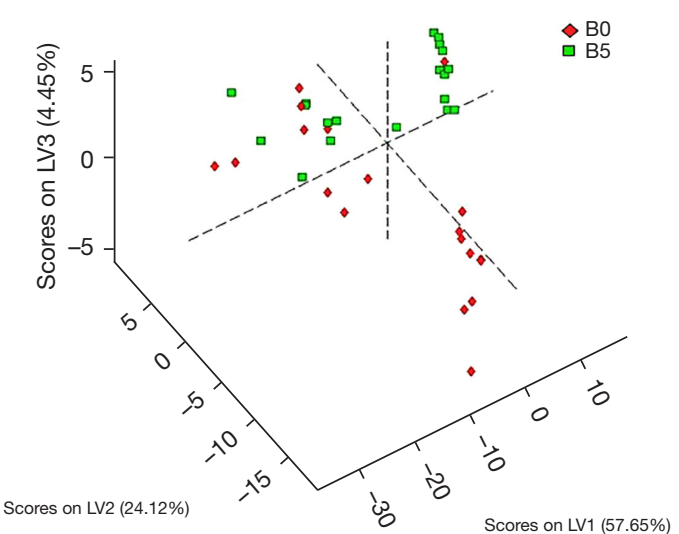

Figure 2 PLS-DA of patients at T0 and T5 (A: $1 \mathrm{~mL} / \mathrm{kg}$; B: $2 \mathrm{~mL} / \mathrm{kg})$. PLS-DA, partial least squares-discriminant analysis.

\section{Discussion}

Critically ill children in pediatric intensive care unit (PICU) often fail to meet caloric target through the preferred enteral route. The result of a systematic review showed (9) that nitrogen balances and inflammation markers, which appeared to be beneficially affected by providing more or altered parenteral nutrition early during critical illness.
However, there are few studies on the early use of parenteral nutrition in PICU. Large RCTs with clinically relevant outcome measures are urgently needed to support the current nutritional guidelines that advise the use of parenteral nutrition in the PICU. Besides, nutrition support via the parenteral route has shown to increase the risk of metabolic disturbances such as hyperglycemia and dyslipidemia and 


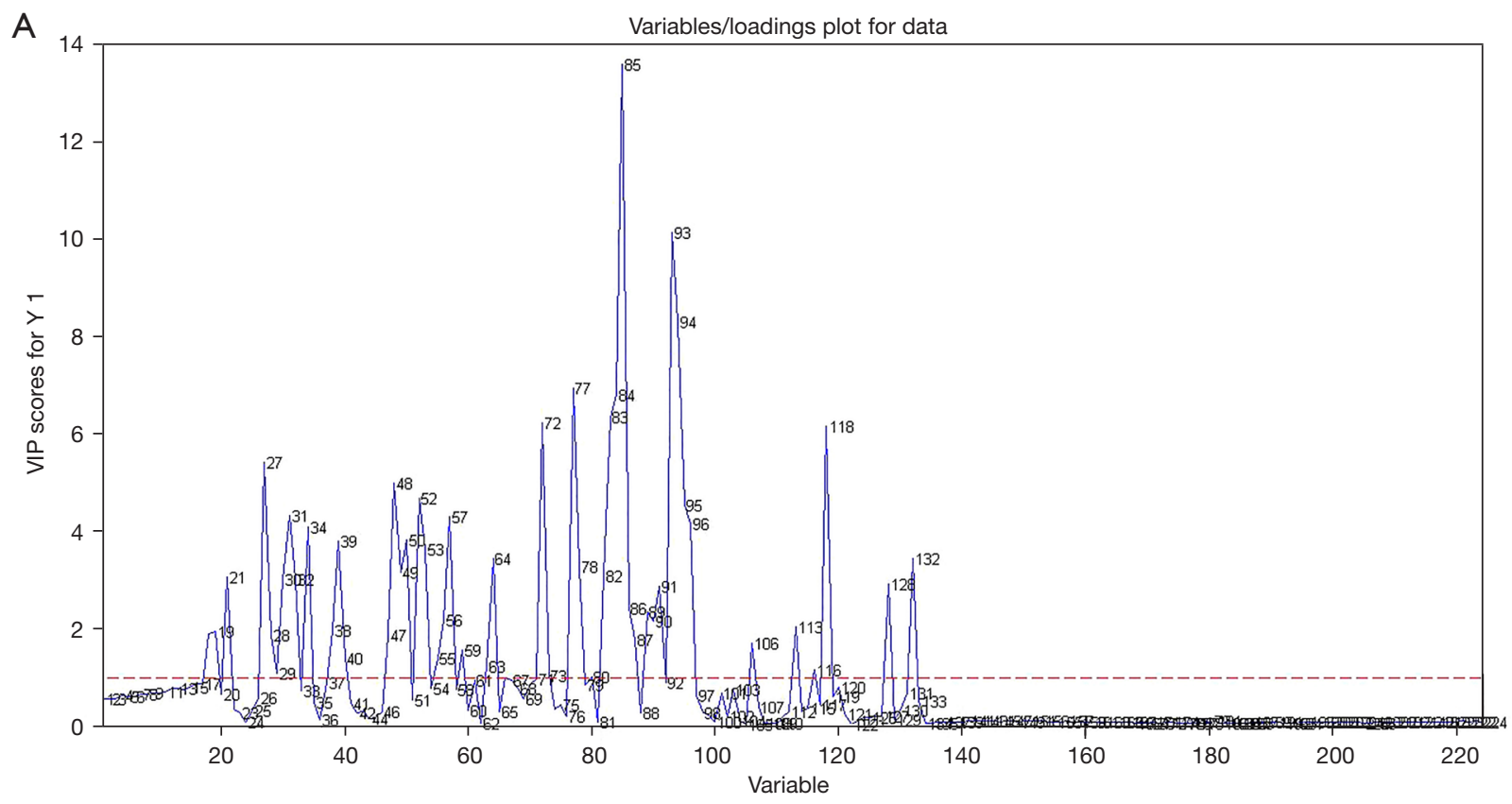

B

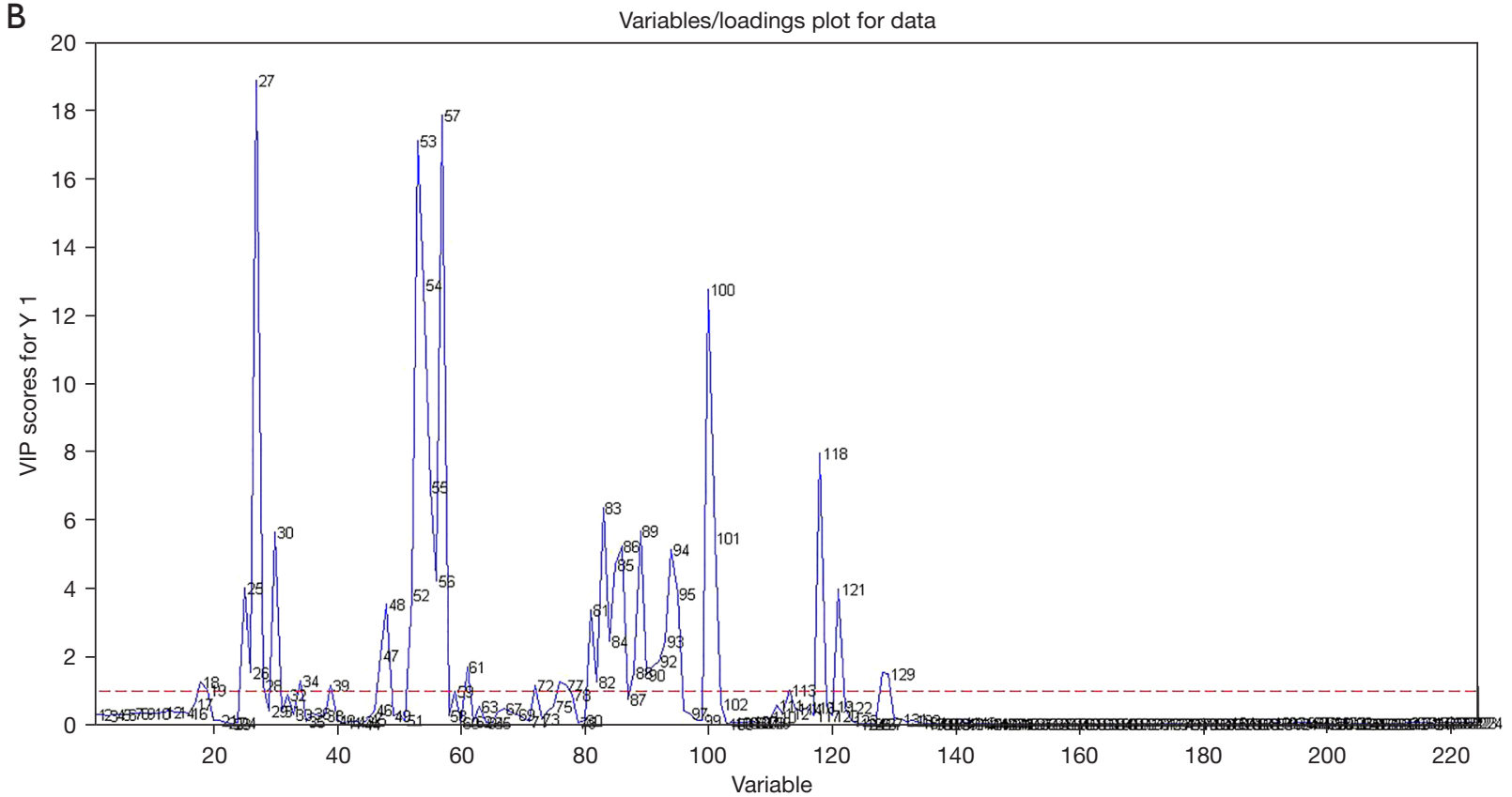

Figure 3 PLS-DA VIP values of chemical shifts between T0 and T5 (A: $1 \mathrm{~mL} / \mathrm{kg} ; \mathrm{B}: 2 \mathrm{~mL} / \mathrm{kg}$ ). PLS-DA, partial least squares-discriminant analysis; VIP, variable importance in the projection.

to be associated with more nosocomial infections (10). According to Guidelines for pediatric clinical application of enteral and parenteral nutritional support in China (2), the route of parenteral nutrition should be established by qualified medical personnel and catheter-related blood stream infections (CRBSI) should be prevented. In addition, pediatric patients receiving long-term parenteral nutrition should supplement with trace elements. Moreover, body composition, blood and biochemical detection should be monitored regularly.

Although TE levels in human tissue account for $<0.01 \%$ of total organism mass, these components are vital for human 
Table 6 Small metabolite differences during patient treatment in Group A $(\mathrm{P}<0.05)$

\begin{tabular}{lccc}
\hline Metabolic pathway & Chemical shift (PPM) & Metabolite & HMDB \\
\hline Valine, leucine, and isoleucine degradation & 1.12 & $\alpha$-ketoisovaleric acid & HMDB0000019 \\
Taurine and hypotaurine metabolism & 3.36 & Hypotaurine & HMDB0000965 \\
Arginine and proline metabolism & 3.40 & Phosphocreatine & HMDB0001511 \\
& 3.76 & Glycocyamine & HMDB0000128 \\
Ketone body metabolism & 3.44 & Acetoacetic acid & HMDB0000060 \\
N/A & 3.80 & Acetyl phosphate & HMDB0031419 \\
\hline
\end{tabular}

PPM, parts per million; HMDB, human metabolome database; N/A, not applicable.

Table 7 Small metabolite differences during patient treatment in Group B $(\mathrm{P}<0.05)$

\begin{tabular}{lccc}
\hline Metabolic pathway & Chemical shift (PPM) & Metabolite & HMDB \\
\hline Valine, leucine and isoleucine degradation & 1.12 & $\alpha$-ketoisovaleric acid & HMDB0000019 \\
Ketone body metabolism & 2.16 & Acetone & HMDB0001659 \\
Pentose phosphate pathway & 2.20 & D-ribose & HMDB0000283 \\
N/A & 2.32 & Dimethylglyoxal & HMDB0003407 \\
Caffeine metabolism & 4.03 & 7-dimethylxanthine & HMDB0001860 \\
\hline
\end{tabular}

PPM, parts per million; HMDB, human metabolome database; N/A, not applicable.

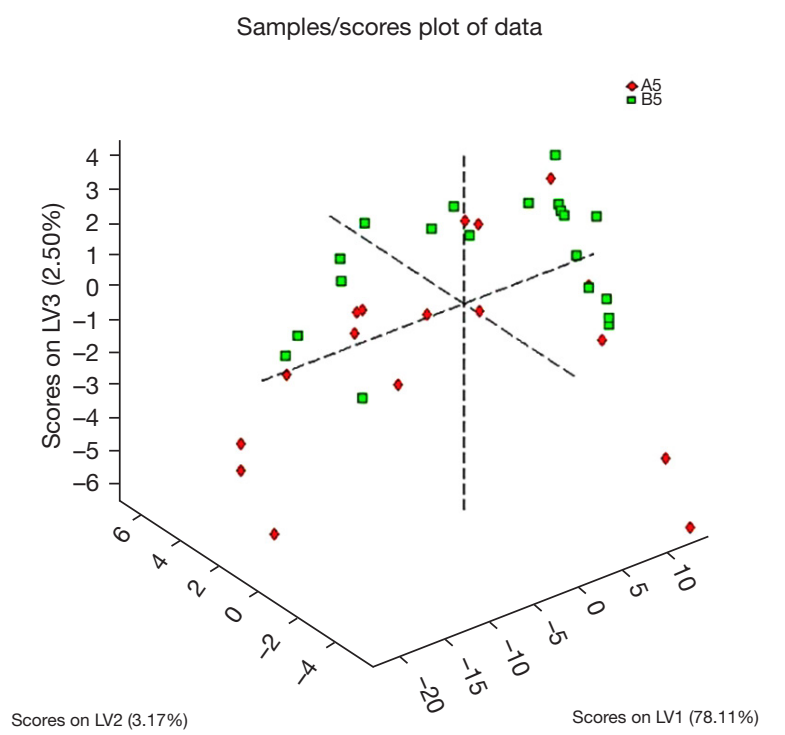

Figure 4 PLS-DA for patients at T5 in Group A and Group B. PLS-DA, partial least squares-discriminant analysis.

growth and development (11). During enteral feeding, patients receive adequate TEs through diversified diets, enteral nutrition products, or oral supplement products. For PN, due to chemical molecule stability, a variety of complex drug products containing multi-TEs are required to meet clinical needs (12). MTEI-(I) is a complex drug product containing multi-TEs specially developed for children. It supplements six trace elements, including $\mathrm{Zn}, \mathrm{Cu}, \mathrm{Mn}, \mathrm{Se}, \mathrm{F}$, and I, but not Fe or chromium, to meet guideline requirements for the addition of TEs during PN $(9,13)$. Guidelines for clinical application of neonatal nutrition support in China (8) recommended that $\mathrm{Zn}$ should be provided with $\mathrm{PN}$ at a dose of $400-500 \mu \mathrm{g} / \mathrm{kg} / \mathrm{d}$ in preterm infants, $250 \mu \mathrm{g} / \mathrm{kg} / \mathrm{d}$ in infants from term to 3 months, $100 \mu \mathrm{g} / \mathrm{kg}$ per day for infants $>3$ months of age; $\mathrm{Cu}$ should be provided with $\mathrm{PN}$ at a dose of $20 \mu \mathrm{g} / \mathrm{kg} /$ day in infants and children; Iodine should be provided with PN at a daily dose of $1 \mu \mathrm{g} / \mathrm{kg}$ daily in infants and children; Se should be provided with $\mathrm{PN}$ at a dose of $2-3 \mu \mathrm{g} / \mathrm{kg} / \mathrm{day}$ in infants and children; $\mathrm{Mn}$ should be supplied in long term PN at a dose of no more than $1 \mu \mathrm{g} / \mathrm{kg} /$ day. European guidelines raise dosage standards for preterm infants (9).

Inflammatory mechanisms generated by inflammation and oxidative stress responses from free radical accumulation often cause normal proteins, lipids, and nucleic acids to attack, undermine, and destroy normal physiological functions. TEs are required for the regulation 


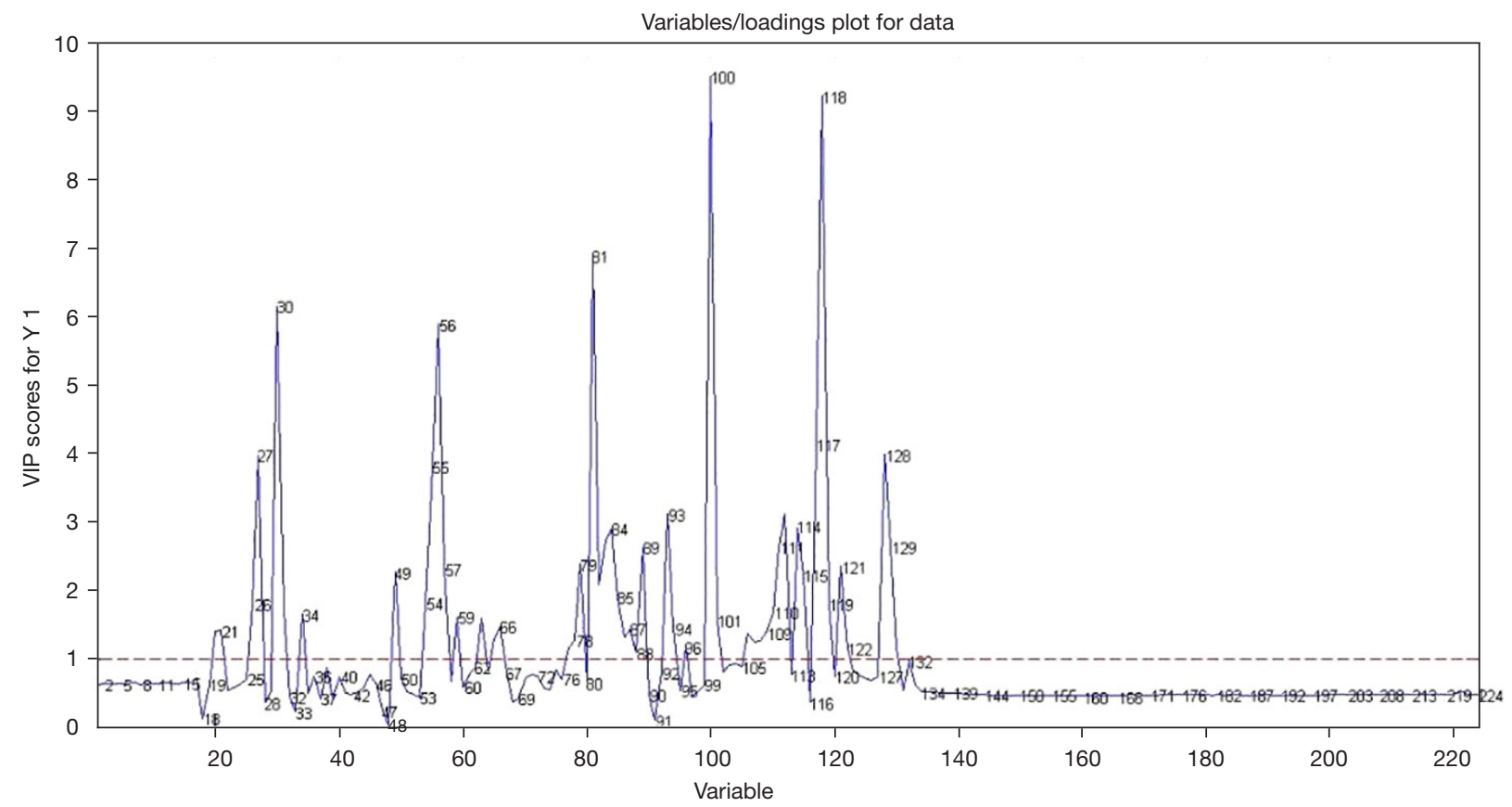

Figure 5 VIP chemical shift values between T5 metabolic differences in Group A and Group B. VIP, variable importance in the projection.

Table 8 Small metabolite differences at T5 treatment in Groups A and B

\begin{tabular}{|c|c|c|c|c|}
\hline Metabolic pathway & Chemical shift (PPM) & Metabolite & HMDB & Trend \\
\hline Arginine and proline metabolism & 1.08 & Phosphocreatine & HMDB0001511 & $\downarrow$ \\
\hline Pentose phosphate metabolism & 2.20 & D-ribose & HMDB0000283 & $\downarrow$ \\
\hline Ketone body metabolism & 2.24 & Acetone & HMDB0001659 & $\downarrow$ \\
\hline Citric acid cycle & 2.40 & Succinic acid & HMDB0000254 & $\downarrow$ \\
\hline Purine metabolism & 3.20 & Adenine & HMDB0000034 & $\downarrow$ \\
\hline Caffeine metabolism & 4.08 & Dimethylxanthine & HMDB0001860 & $\downarrow$ \\
\hline $\mathrm{N} / \mathrm{A}$ & 4.40 & Dihydroxy acetone & HMDB0001882 & $\downarrow$ \\
\hline
\end{tabular}

PPM, parts per million; HMDB, human metabolome database; N/A, not applicable.

of substance metabolism, enzyme catalytic activity, etc., and thus affect inflammation and oxidative stress mediators. For example, during oxidative stress and inflammation, TE distribution will be altered, and thus, a reasonable intake of these elements will exert positive effects towards inflammation control, and slow or reduce oxidative stress responses (14). Previous studies have demonstrated that appropriate $\mathrm{Cu}, \mathrm{Zn}$, and Se levels reduce free radicals, enhance antioxidant capacity, and regulate inflammatory reactions (15-17). Meanwhile, $\mathrm{Cu}$ is positively correlated with bacterial levels and inflammatory markers $(18,19)$, while $\mathrm{Zn}$ and $\mathrm{Se}$ are negatively correlated with inflammation and oxidative stress (20,21). Supplementation with $\mathrm{Zn}$ has been shown to improve high $\mathrm{Zn}-\mathrm{Cu}$ ratios in the blood, 
reduce oxidative stress, improve inflammatory conditions, and maintain immune functions (22). These previous reports are consistent with our findings, which indicated that $\mathrm{Cu}$ was decreased, and $\mathrm{Zn}$ and Se were increased after PN treatment, with TE differences in Group B being more significant. Equally, we observed that WBC levels in both groups were decreased after PN treatment, with levels in Group B being significantly decreased after PN treatment $(\mathrm{P}=0.011)$. This observation suggests that the appropriate high-dose administration of MTEI-(I) was effectively controlling inflammation and antioxidation.

Hexacosanoic acid is a very-long-chain fatty acid, and is an important component of phospholipid molecules. A previous study (23) found that these molecules play important roles in cellular biochemical reactions, nutrient storage, and intercellular communications. Due to homeostatic imbalances between molecular transport and utilization, excessive fatty acid accumulation may cause toxicity in some tissues, which becomes manifested as oxidative stress and inflammation, potentially culminating in cell apoptosis (24). Several studies have reported that verylong-chain fatty acids induce the production of reactive oxygen species in the SK-N-BE neuroblastoma cell line, and enhance oxidative stress (25). Dhaunsi et al. (26) observed these molecules activate nicotinamide adenine dinucleotide phosphate oxidase activity, and enhance superoxide anionmediated lipid peroxidation in skin fibroblasts. In this study, we observed that the $\beta$-oxidation of very-long-chain fatty acids (hexacosanoic acid) was significantly reduced in Group B $(\mathrm{P}<0.05)$, indicating patients were less prone to oxidative damage caused by lipid peroxidation. Therefore, appropriate high-dose administration of MTEI-(I) exerted positive antioxidation effects in this group (27).

Stress has an important impact on various metabolic pathways. Under stress conditions, the following metabolic characteristics are often observed: high metabolic rate, increased catabolism, and reduced anabolic metabolism, resulting in a negative balance in overall metabolism. In this study, 37 children were under acute stress after surgery or disease. Chen et al. (28) observed that stress induces the loss of $\mathrm{Zn}$ from the body, and $\mathrm{Zn}$ supplementation exerts protective effects. In this study, after supplementing MTEI(I), we observed that valine, leucine, isoleucine degradation, taurine and hypotaurine metabolism, arginine and proline metabolism, and other amino acid metabolism were all reduced, suggesting a benefit to disease recovery. At the same time, ketone metabolism was also reduced, suggesting that the high metabolic rate had been relieved. Of these components, $\beta$-oxidation of very-long-chain fatty acids, pentose phosphate metabolism, ketone body metabolism, citric acid cycle, and pyruvate metabolism were all significantly reduced in Group B. These factors were related to energy metabolism $(29,30)$, indicating that appropriate high-dose administration of MTEI-(I) was helpful in relieving stress-induced elevated metabolism.

Hypoxia is a basic pathological process that is implicated in several diseases (31). Severe hypoxia induces considerable cellular harm, and often leads to death. Kim et al. (32) observed that $\mathrm{Zn}$ ameliorates hypoxic neuronal death induced by deferoxamine (DFX) and sodium azide $\left(\mathrm{NaN}_{3}\right)$. $\mathrm{Yu}$ et al. (33) reported that $\mathrm{Zn}$ chelating agents had protective effects towards hypoxic ischemic brain damage in zebrafish. Xie et al. (34) proposed that exogenous $\mathrm{Zn}$ had protective effects towards hypoxic neurons. Hypoxanthine is a naturally occurring purine derivative, and is the major catabolite of adenosine triphosphate (ATP) in hypoxic or ischemic tissue (35). In general terms, a large increase in hypoxanthine levels in bodily fluids indicates adenosine triphosphate (ATP) depletion (36). In a trial of patients with critical illness, burns, and burn-induced sepsis (36), the evidence suggested that elevated ATP-associated degradation products i.e., adenosine, inosine, hypoxanthine, and xanthine were associated with tissue hypo-perfusion and hypoxia levels. Therefore, it was suggested that purine metabolites, such as xanthine and hypoxanthine, are potential markers of tissue hypoxia (37). In our study, the administration of MTEI-(I) in Group B significantly increased plasma Zn levels. In our metabolomics study, we observed that purine metabolism in Group B was significantly reduced, and related metabolites were similarly reduced, indicating that appropriate high-dose administration of MTEI-(I) improved hypoxic conditions in these patients.

There were several limitations in this study that should be noted. Firstly, the sample size was relatively small, especially the disease spectrum is relatively single. It is difficult to reveal the effect of parenteral nutrition on serum free trace elements in patients with different diseases in this study. More samples with different diseases should be included in future studies. Also, this was a single center study, and thus, multi-center studies are needed to verify our conclusions.

\section{Conclusions}

In summary, the high-dose administration of MTEI-(I) is beneficial for pediatric patients. Such administration does 
not increase the burden on visceral organs, and appears to exert protective effects on liver and kidney functions. Several studies have shown that Se protects the kidney from oxidative damage, and reduces oxidative damage to the kidney (38-40). The high-dose administration of MTEI(I) also reduces serum glutamic pyruvic transaminase, total and direct bilirubin, and has been shown to reduce ultrastructure liver cell damage in rats (41). Supplementation with $\mathrm{Zn}$ also delays the progression of chronic kidney disease damage and relieves its complications $(42,43)$. In this study, $\mathrm{Zn}$ and Se plasma levels were increased by MTEI(I) administration. Also, liver and kidney functional analyses of our patients indicated that $\mathrm{Cr}, \mathrm{TB}, \mathrm{DB}$, and ALB levels were decreased after supplementation with MTEI-(I), and Cr was significantly decreased in Group B, suggesting that appropriate high-dose supplementation of MTEI-(I) was beneficial in improving renal function. Finally, the economic aspects and duration in hospital were also considered in this study, and administration at a high dose did not increase expenditure and duration dramatically.

\section{Acknowledgments}

We sincerely acknowledge and appreciate the support from Beijing Qingxi Technology Research Institute.

Funding: This work was supported by the Sichuan Science and Technology Program (grants ID 2019YFS0303, 2019YFS0534 and 2020YFS0392) and the Key project of Sichuan Provincial Health Commission (grants ID 209J102). The funders played no role in the study search, selection, and analyses that were conducted by researchers.

\section{Footnote}

Reporting Checklist: The authors have completed the CONSORT reporting checklist. Available at https://dx.doi. org/10.21037/tp-21-456

Data Sharing Statement: Available at https://dx.doi. org/10.21037/tp-21-456

Trial Protocol: Available at https://dx.doi.org/10.21037/tp$21-456$

Conflicts of Interest: All authors have completed the ICMJE uniform disclosure form (available at https://dx.doi. org/10.21037/tp-21-456). The authors have no conflicts of interest to declare.
Ethical Statement: The authors are accountable for all aspects of the work in ensuring that questions related to the accuracy or integrity of any part of the work are appropriately investigated and resolved. The study was conducted according to the guidelines of the Declaration of Helsinki (as revised in 2013) and approved by the ethics committee of Chengdu Women's and Children's Central Hospital (No.2017 [21]). All patients or statutory guardians provided informed consent.

Open Access Statement: This is an Open Access article distributed in accordance with the Creative Commons Attribution-NonCommercial-NoDerivs 4.0 International License (CC BY-NC-ND 4.0), which permits the noncommercial replication and distribution of the article with the strict proviso that no changes or edits are made and the original work is properly cited (including links to both the formal publication through the relevant DOI and the license). See: https://creativecommons.org/licenses/by-nc-nd/4.0/.

\section{References}

1. Jacobs A, Verlinden I, Vanhorebeek I, et al. Early Supplemental Parenteral Nutrition in Critically Ill Children: An Update. J Clin Med 2019;8:830.

2. Pediatric Collaborative Group, Society of Parenteral and Enteral Nutrition, Chinese Medical Association. Guidelines for pediatric clinical application of enteral and parenteral nutritional support in China. Zhonghua Er Ke Za Zhi 2010;48:436-41.

3. Tabor E. Current Status of Multi-Trace Element Products for Parenteral Nutrition in the United States. Nutr Clin Pract 2019;34:487-8.

4. Marino LV, Valla FV, Beattie RM, et al. Micronutrient status during paediatric critical illness: A scoping review. Clin Nutr 2020;39:3571-93.

5. Shenkin A. Micronutrients in health and disease. Postgrad Med J 2006;82:559-67.

6. Wishart DS. Metabolomics for Investigating Physiological and Pathophysiological Processes. Physiol Rev 2019;99:1819-75.

7. de Jesus JR, de Araújo Andrade T. Understanding the relationship between viral infections and trace elements from a metallomics perspective: implications for COVID-19. Metallomics 2020;12:1912-30.

8. Cai W, Tang QY, Wang Y, et al. Guidelines for clinical application of neonatal nutrition support in China. J Clin Pediatr 2013;31:1177-82. 
9. Domellöf M, Szitanyi P, Simchowitz V, et al. ESPGHAN/ ESPEN/ESPR/CSPEN guidelines on pediatric parenteral nutrition: Iron and trace minerals. Clin Nutr 2018;37:2354-9.

10. Fivez T, Kerklaan D, Mesotten D, et al. Evidence for the use of parenteral nutrition in the pediatric intensive care unit. Clin Nutr 2017;36:218-23.

11. Stehle P, Stoffel-Wagner B, Kuhn KS. Parenteral trace element provision: recent clinical research and practical conclusions. Eur J Clin Nutr 2016;70:886-93.

12. Osland EJ, Ali A, Isenring E, et al. Australasian Society for Parenteral and Enteral Nutrition guidelines for supplementation of trace elements during parenteral nutrition. Asia Pac J Clin Nutr 2014;23:545-54.

13. Chinese Society for Parenteral and Enteral Nutrition. The expert consensus on clinical application of multi-trace elements. Zhonghua Wai Ke Za Zhi 2018;56:168-75.

14. Heyland DK, Dhaliwal R, Suchner U, et al. Antioxidant nutrients: a systematic review of trace elements and vitamins in the critically ill patient. Intensive Care Med 2005;31:327-37.

15. Li Y, Zhou C, Wei A. Effect of Trace Element Zinc on Defending Free Radical Damage. Guangdong Trace Elements Science 2001;7:3-5.

16. Wei A, Zhou C. Effect of Trace Element Selenium in Defending Free Radical Damage. Guangdong Trace Elements Science 2001;8:23-5.

17. Tenekeci G, Bilen BT, Turkoz Y, et al. The Effect of Selenium on Ischemia-Reperfusion Injury: An Experimental Study on a Transverse Rectus Abdominis Musculocutaneous Flap Model. J Craniofac Surg 2016;27:242-6.

18. Pompilio A, Ciavardelli D, Crocetta V, et al. Stenotrophomonas maltophilia virulence and specific variations in trace elements during acute lung infection: implications in cystic fibrosis. PLoS One 2014;9:e88769.

19. Shike M. Copper in parenteral nutrition. Gastroenterology 2009;137:S13-7.

20. Cao Q, Jia Z. Correlation Between Serum Trace Element Changes and Levels of Inflammatory Cytokines and Oxidative Stress Indexes in Patients with Urinary Tract Infection. Journal of Hainan Medical College 2018;24:1728-36.

21. Guo $\mathrm{CH}$, Chen PC, Yeh MS, et al. $\mathrm{Cu} / \mathrm{Zn}$ ratios are associated with nutritional status, oxidative stress, inflammation, and immune abnormalities in patients on peritoneal dialysis. Clin Biochem 2011;44:275-80.

22. Guo CH, Wang CL. Effects of zinc supplementation on plasma copper/zinc ratios, oxidative stress, and immunological status in hemodialysis patients. Int J Med Sci 2013;10:79-89.

23. Paz Ramos A, Lagüe P, Lamoureux G, et al. Effect of Saturated Very Long-Chain Fatty Acids on the Organization of Lipid Membranes: A Study Combining (2)H NMR Spectroscopy and Molecular Dynamics Simulations. J Phys Chem B 2016;120:6951-60.

24. Savary S, Trompier D, Andréoletti P, et al. Fatty acids induced lipotoxicity and inflammation. Curr Drug Metab 2012;13:1358-70.

25. Zarrouk A, Vejux A, Nury T, et al. Induction of mitochondrial changes associated with oxidative stress on very long chain fatty acids (C22:0, C24:0, or C26:0)treated human neuronal cells (SK-NB-E). Oxid Med Cell Longev 2012;2012:623257.

26. Dhaunsi GS, Kaur J, Alsaeid K, et al. Very long chain fatty acids activate NADPH oxidase in human dermal fibroblasts. Cell Biochem Funct 2005;23:65-8.

27. Shin TH, Kim HA, Jung JY, et al. Analysis of the free fatty acid metabolome in the plasma of patients with systemic lupus erythematosus and fever. Metabolomics 2017;14:14.

28. Chen WQ, Cheng YY, LI ST, et al. Effects of Zinc on the Expression of Metallothionein Isoforms in Different Brain Domains in Rats Under Stress. Acta Nutrimenta Sinica 2006;28:288-91.

29. Stincone A, Prigione A, Cramer T, et al. The return of metabolism: biochemistry and physiology of the pentose phosphate pathway. Biol Rev Camb Philos Soc 2015;90:927-63.

30. Wu X, Guo J, Chen Y, et al. The 60-kDa heat shock protein regulates energy rearrangement and protein synthesis to promote proliferation of multiple myeloma cells. Br J Haematol 2020;190:741-52.

31. Lee JW, Ko J, Ju C, et al. Hypoxia signaling in human diseases and therapeutic targets. Exp Mol Med 2019;51:1-13.

32. Kim S, Seo JW, Oh SB, et al. Disparate roles of zinc in chemical hypoxia-induced neuronal death. Front Cell Neurosci 2015;9:1.

33. Yu X, Li YV. Neuroprotective effect of zinc chelator DEDTC in a zebrafish (Danio rerio) Model of Hypoxic Brain Injury. Zebrafish 2013;10:30-5.

34. Xie K, Ju C, Min H, et al. Protective effect of exogenous zinc on neurons from hypoxic injury. Chinese Journal of Anatomy 2008;31:684-61.

35. Farthing DE, Farthing CA, Xi L. Inosine and 
hypoxanthine as novel biomarkers for cardiac ischemia: from bench to point-of-care. Exp Biol Med (Maywood) 2015;240:821-31.

36. Liu XR, Zheng XF, Ji SZ, et al. Metabolomic analysis of thermally injured and/or septic rats. Burns 2010;36:992-8.

37. Mogensen KM, Lasky-Su J, Rogers AJ, et al. Metabolites Associated With Malnutrition in the Intensive Care Unit Are Also Associated With 28-Day Mortality. JPEN J Parenter Enteral Nutr 2017;41:188-97.

38. Diyabalanage S, Fonseka S, Dasanayake DMSNB, et al. Environmental exposures of trace elements assessed using keratinized matrices from patients with chronic kidney diseases of uncertain etiology (CKDu) in Sri Lanka. J Trace Elem Med Biol 2017;39:62-70.

39. Wiesen $P$, Van Overmeire L, Delanaye P, et al. Nutrition disorders during acute renal failure and renal replacement therapy. JPEN J Parenter Enteral Nutr 2011;35:217-22.

40. Iglesias P, Selgas R, Romero S, et al. Selenium and kidney disease. J Nephrol 2013;26:266-72.

41. Wang L. Trace Elements Selenium to Obstruction Jaundice Liver Cytoprotection Research. Journal of Liaoning Medical University 2010;3:21-2.

42. Hadj Abdallah N, Baulies A, Bouhlel A, et al. Zinc mitigates renal ischemia-reperfusion injury in rats by modulating oxidative stress, endoplasmic reticulum stress, and autophagy. J Cell Physiol 2018;233:8677-90.

43. Iorember FM. Malnutrition in Chronic Kidney Disease. Front Pediatr 2018;6:161.

(English Language Editor: A. Kassem)
Cite this article as: Tan Q, Wang Y, Zhang G, Lu B, Wang T, Tao T, Wang H, Jiang H, Chen W. The metabolic effects of multi-trace elements on parenteral nutrition for critically ill pediatric patients: a randomized controlled trial and metabolomic research. Transl Pediatr 2021;10(10):2579-2593. doi: $10.21037 / \mathrm{tp}-21-456$ 